\title{
Rural Residency Curricula: Potential Target for Improved Access to Care?
}

\author{
Alexandra Streifel, BA; Laurel L. Wessman, MD; Ronda S. Farah, MD; Kevin J. Gaddis, MD; \\ Adam Byrd, MD; Robert T. Brodell, MD; Cindy Firkins Smith, MD
}

\section{PRACTICE POINTS}

- Access to dermatologic care in rural areas is a growing problem.

- Dermatology residency programs can influence medical students and resident dermatologists to provide care in rural and geographically isolated areas.

- Presenting detailed curricula that impact access to care on residency program websites could attract applicants with these career goals.

To the Editor:

There is an irrefutable trend toward urban dermatology practice in the United States, leading to growing problems with rural access to care. The provision of rural clinical experiences and telehealth in dermatology residency training might increase the likelihood of trainees establishing a rural practice.

In 2017, the American Academy of Dermatology released an updated statement supporting direct patient access to board-certified dermatologists in an effort to reduce morbidity and mortality associated with skin disease. ${ }^{1}$ Twenty percent of the US population lives in a rural and medically underserved location, yet these areas remain largely underserved, in part because of an irrefutable trend toward urban dermatology practice..$^{2-4}$ Successful approaches to improving rural access to dermatology care are poorly defined in the literature.

Several variables have been shown to influence a young physician's decision to establish a clinical practice in geographically isolated areas, including rural upbringing, longitudinal rural clinical experiences during medical training, and family influences. ${ }^{5}$ Location of residency training is an additional variable that impacts practice location, though migration following dermatology residency is a complex phenomenon. However, training location does not guarantee retention of dermatology graduates in any particular geographic area. ${ }^{6}$ Practice incentives and stipends might encourage rural dermatology practice, yet these programs are underfunded. Last, telemedicine in dermatology (including teledermatology and teledermoscopy), though not always an ideal substitute for a live visit, can improve access to care in geographically isolated or underserved areas in general. ${ }^{7-9}$

Focused recruitment of medical students interested in rural dermatology practice to accredited dermatology residency programs aligned with this goal represents another approach to improve geographic diversity in the field of dermatology. Online access to this information would be useful for both applicants and their mentors.

We assessed viewable online curricula related to rural dermatology and telemedicine experiences at all Accreditation Council for Graduate Medical Education (ACGME)-accredited residency programs. Telemedicine experiences at Veterans Health Administration (VHA) health systems also were assessed.

\section{Methods}

This study was exempt from review by the institutional review board at the University of Minnesota (Minneapolis, Minnesota)(IRB \#STUDY00004915) because no human subjects were involved. Online curricula of all ACGMEaccredited dermatology residency programs in the United States and Puerto Rico were reviewed from November to December 2018. The following information was recorded: specialized "rural-track" training; optional elective time in rural settings; teledermatology training; and teledermoscopy training.

\footnotetext{
Ms. Streifel is from the University of North Dakota School of Medicine, Grand Forks. Drs. Wessman, Farah, and Gaddis are from the Department of Dermatology, University of Minnesota, Minneapolis. Drs. Byrd and Brodell are from the Department of Dermatology, University of Mississippi Medical Center, Jackson. Dr. Smith is from Carris Health, Willmar, Minnesota.

The authors report no conflict of interest.

The eTable is available in the Appendix online at www.mdedge.com/dermatology.

Correspondence: Cindy Firkins Smith, MD, Carris Health, 101 Willmar Ave SW, Willmar, MN 56201 (cindy.smith@carrishealth.com). doi:10.12788/cutis.0149
} 
Additionally, population density at each program's primary location was determined using US Census Bureau data and with consideration to communities contained within particular Metropolitan Statistical Areas (MSAs)(eTable). Data were obtained from the VHA system to assess teledermatology services at VHA locations affiliated with residency programs.

\section{Results}

Of 154 dermatology residency programs identified in the United States and Puerto Rico, 142 were accredited at the time of data collection. Fifteen (10\%) were based in communities of 50,000 individuals or fewer that were not near a large metropolitan area. One program $(<1 \%)$ offered a specific rural track. Fifty-six programs (39\%) cited optional rotations or clinical electives, or both, that could be utilized for a rural experience. Eighteen (12\%) offered teledermatology experiences and $1(<1 \%)$ offered teledermoscopy during training. Fifty-three programs (37\%) offered a rotation at a VHA hospital that had an active teledermatology service.

\section{Comment}

Program websites are a free and easily accessible means of acquiring relevant information. The paucity of readily available data on rural dermatology and teledermatology opportunities is unfortunate and a detriment to dermatology residency applicants interested in rural practice, which may result in a missed opportunity to foster a true passion for rural medicine. A brief comment on a website can be impactful, leading to a postgraduate year 4 dermatology elective rotation at a prospective fellowship training site or a rural dermatology experience.

The paucity of dermatologists working directly in rural areas has led to development of teledermatology initiatives to reach deeply into underserved regions. One of the largest providers of teledermatology is the VHA, which standardized its teledermatology efforts in 2012 and provides remarkable educational opportunities for dermatology residents. However, many residency program and VHA websites provide no information about the participation of dermatology residents in the provision of teledermatology services.
A limitation of this study is that it is based on online published curricula. Dermatology residency programs with excellent rural curricula that are not published online might exist.

Residency program directors with an interest in geographic diversity are encouraged to provide rural and teledermatology opportunities and to update these offerings on their websites, which is a simple modifiable strategy that can impact the rural dermatology care gap by recruiting students interested in filling this role. These efforts should be studied to determine whether this strategy impacts resident selection as well as whether focused rural and telemedicine exposure during training increases the likelihood of establishing a rural dermatology practice in the future.

\section{REFERENCES}

1. American Academy of Dermatology. Position statement on access to specialty care and direct access to dermatologic care. Revised May 20, 2017. Accessed December 13, 2020. https://server.aad.org /forms/Policies/Uploads/PS/PS - Access \% 20 to \% 20Specialty $\% 20$ Care $\% 20$ and $\% 20$ Direct $\% 20$ Access $\% 20$ to $\% 20$ Dermatologic \%20Care.pdf

2. Dill MJ, Salsberg ES. The Complexities of Physician Supply and Demand: Projections Through 2025. Center for Workforce Studies, Association of American Medical Colleges (AAMC); November 2008. Accessed December 13, 2020. http://innovationlabs.com/pa_future/1 /background_docs/AAMC \%20Complexities \%20of\%20physician $\% 20$ demand, \%202008.pdf

3. Glazer AM, Rigel DS. Analysis of trends in geographic distribution of US dermatology workforce density. JAMA Dermatol. 2017;153:472-473.

4. Yoo JY, Rigel DS. Trends in dermatology: geographic density of US dermatologists. Arch Dermatol. 2010;146:779.

5. Feng H, Berk-Krauss J, Feng PW, et al. Comparison of dermatologist density between urban and rural counties in the United States. JAMA Dermatol. 2018;154:1265-1271.

6. Landow SM, Oh DH, Weinstock MA. Teledermatology within the Veterans Health Administration, 2002-2014. Telemed J E Health. 2015;21:769-773.

7. Armstrong AW, Kwong MW, Ledo L, et al. Practice models and challenges in teledermatology: a study of collective experiences from teledermatologists. PloS One. 2011;6:e28687.

8. Lewis H, Becevic M, Myers D, et al. Dermatology ECHO_an innovative solution to address limited access to dermatology expertise. Rural Remote Health. 2018;18:4415.

9. Edison KE, Dyer JA, Whited JD, et al. Practice gaps. the barriers and the promise of teledermatology. JAMA Dermatol. 2012:148:650-651. 


\section{APPENDIX}

eTABLE. Comparison of Viewable Online Curricula of ACGME-Accredited Dermatology Residency Programs by Rural Track Training, Rural Clinical Rotation Opportunities, and Experience in Teledermatology/Teledermoscopy ${ }^{\mathrm{a}}$

\begin{tabular}{|c|c|c|c|c|c|c|}
\hline Program name & City & State & $\begin{array}{l}\text { Population }^{b} \\
\text { (associated } \\
\text { metropolitan area, } \\
\text { if applicable) }\end{array}$ & $\begin{array}{l}\text { Rural } \\
\text { track }\end{array}$ & $\begin{array}{l}\text { Optional } \\
\text { elective } \\
\text { opportunity }\end{array}$ & Teledermatology \\
\hline $\begin{array}{l}\text { University of Arkansas for } \\
\text { Medical Sciences }\end{array}$ & Little Rock & AR & 198,606 & $\mathrm{~N}$ & Y & $\mathrm{N}$ \\
\hline $\begin{array}{l}\text { Mayo Clinic College of Medicine } \\
\text { and Science (Arizona) }\end{array}$ & Scottsdale & $A Z$ & $\begin{array}{l}249,950 \\
(1,455,632)\end{array}$ & $\mathrm{N}$ & Y & $\mathrm{N}$ \\
\hline $\begin{array}{l}\text { Midwestern University Osteopathic } \\
\text { Postdoctoral Training Institute }\end{array}$ & Peoria & $A Z$ & $\begin{array}{l}168,181 \\
(1,455,632)\end{array}$ & $\mathrm{N}$ & $Y$ & $\mathrm{~N}$ \\
\hline $\begin{array}{l}\text { Kaiser Permanente Southern } \\
\text { California (Los Angeles) }\end{array}$ & Los Angeles & CA & $3,792,621$ & & Y & $\mathrm{N}$ \\
\hline $\begin{array}{l}\text { Los Angeles County-Harbor-UCLA } \\
\text { Medical Center }\end{array}$ & Torrance & CA & $\begin{array}{l}146,758 \\
(3,792,621)\end{array}$ & $\mathrm{N}$ & Y & $\mathrm{N}$ \\
\hline OPTI West (Newport Beach) Program & $\begin{array}{l}\text { Newport } \\
\text { Beach }\end{array}$ & CA & & $\mathrm{N}$ & Y & $\mathrm{N}$ \\
\hline $\begin{array}{l}\text { Stanford Health Care-Sponsored } \\
\text { Stanford University }\end{array}$ & $\begin{array}{l}\text { Redwood } \\
\text { City }\end{array}$ & $\mathrm{CA}$ & 86,685 & $\mathrm{~N}$ & Y & $\mathrm{N}$ \\
\hline $\begin{array}{l}\text { UCLA David Geffen School of } \\
\text { Medicine/UCLA Medical Center }\end{array}$ & Los Angeles & & $3,792,621$ & $\mathrm{~N}$ & Y & $\mathrm{N}$ \\
\hline University of California Davis Health & Sacramento & CA & 501,901 & $\mathrm{~N}$ & Y & Y \\
\hline $\begin{array}{l}\text { University of California (San Diego) } \\
\text { Medical Center }\end{array}$ & $\begin{array}{l}\text { La Jolla/San } \\
\text { Diego }\end{array}$ & CA & $46,000(1,400,000)$ & $\mathrm{N}$ & Y & $\mathrm{N}$ \\
\hline $\begin{array}{l}\text { University of California } \\
\text { (San Francisco) }\end{array}$ & $\begin{array}{l}\text { San } \\
\text { Francisco }\end{array}$ & CA & 884,363 & $\mathrm{~N}$ & Y & Y \\
\hline $\begin{array}{l}\text { University of Southern California/ } \\
\text { LAC+USC Medical Center }\end{array}$ & Los Angeles & $\mathrm{CA}$ & $3,792,621$ & $\mathrm{~N}$ & $\mathrm{~N}$ & Y \\
\hline University of Colorado & Aurora & $\mathrm{CO}$ & $366,623(600,158)$ & $\mathrm{N}$ & $\mathrm{N}$ & Y \\
\hline University of Connecticut & Farmington & CT & 25,340 & $\mathrm{~N}$ & Y & $\mathrm{N}$ \\
\hline Yale-New Haven Medical Center & New Haven & CT & 131,014 & $\mathrm{~N}$ & Y & $\mathrm{N}$ \\
\hline Howard University & Washington & DC & 693,972 & $\mathrm{~N}$ & Y & $\mathrm{N}$ \\
\hline $\begin{array}{l}\text { MedStar Heath/Washington } \\
\text { Hospital Center }\end{array}$ & Washington & $\mathrm{DC}$ & 693,972 & $\mathrm{~N}$ & Y & $\mathrm{N}$ \\
\hline $\begin{array}{l}\text { HCA West Florida GME Consortium/ } \\
\text { Largo Medical Center }\end{array}$ & Largo & FL & 84,754 & $\mathrm{~N}$ & Y & $\mathrm{N}$ \\
\hline $\begin{array}{l}\text { Mayo Clinic College of Medicine and } \\
\text { Science (Jacksonville) }\end{array}$ & Jacksonville & $\mathrm{FL}$ & 892,062 & $\mathrm{~N}$ & Y & $\mathrm{N}$ \\
\hline
\end{tabular}


eTABLE (CONTINUED)

\begin{tabular}{|c|c|c|c|c|c|c|}
\hline Program name & City & State & $\begin{array}{l}\text { Population }^{\text {b }} \\
\text { (associated } \\
\text { metropolitan area, } \\
\text { if applicable) }\end{array}$ & $\begin{array}{l}\text { Rural } \\
\text { track }\end{array}$ & $\begin{array}{l}\text { Optional } \\
\text { elective } \\
\text { opportunity }\end{array}$ & Teledermatology \\
\hline Orange Park Medical Center & Orange Park & FL & $8705(821,784)$ & $\mathrm{N}$ & Y & $\mathrm{N}$ \\
\hline University of Florida & Gainesville & $\mathrm{FL}$ & 132,249 & $\mathrm{~N}$ & Y & $\mathrm{N}$ \\
\hline Emory University School of Medicine & Atlanta & GA & 486,290 & $\mathrm{~N}$ & $\mathrm{~N}$ & Y \\
\hline $\begin{array}{l}\text { Philadelphia College of Osteopathic } \\
\text { Medicine }\end{array}$ & Roswell & GA & 94,786 & $\mathrm{~N}$ & Y & $\mathrm{N}$ \\
\hline $\begin{array}{l}\text { University of lowa Hospitals } \\
\text { and Clinics }\end{array}$ & lowa City & IA & 75,798 & $\mathrm{~N}$ & Y & $\mathrm{N}$ \\
\hline Loyola University Medical Center & Maywood & IL & $25,090(2,716,450)$ & $\mathrm{N}$ & & $\mathrm{N}$ \\
\hline $\begin{array}{l}\text { McGaw Medical Center of } \\
\text { Northwestern University }\end{array}$ & Chicago & IL & $2,716,450$ & N & & $\mathrm{N}$ \\
\hline Southern Illinois University & Springfield & IL & 114,868 & $\mathrm{~N}$ & Y & $\mathrm{N}$ \\
\hline University of Chicago & Chicago & IL & $2,716,450$ & $\mathrm{~N}$ & Y & $\mathrm{N}$ \\
\hline $\begin{array}{l}\text { University of Louisville School } \\
\text { of Medicine }\end{array}$ & Louisville & KY & 621,349 & $\mathrm{~N}$ & Y & $\mathrm{N}$ \\
\hline Boston University Medical Center & Boston & MA & 685,094 & $\mathrm{~N}$ & $\mathrm{~N}$ & Y \\
\hline $\begin{array}{l}\text { MA General Hospital/Beth Israel } \\
\text { Deaconess Medical Center/Brigham } \\
\text { and Women's }\end{array}$ & Boston & & 685,094 & $\mathrm{~N}$ & Y & Y \\
\hline Tufts Medical Center & Boston & MA & 685,094 & $\mathrm{~N}$ & Y & $Y^{c}$ \\
\hline University of Maryland & & MD & 611,648 & $\mathrm{~N}$ & Y & $\mathrm{N}$ \\
\hline St Joseph Mercy Livingston & Ann Arbor & Ml & 121,477 & $\mathrm{~N}$ & Y & $\mathrm{N}$ \\
\hline University of Michigan Health System & Ann Arbor & Ml & 121,477 & $\mathrm{~N}$ & $\mathrm{~N}$ & Y \\
\hline $\begin{array}{l}\text { Mayo Clinic College of Medicine } \\
\text { and Science (Rochester) }\end{array}$ & Rochester & $\mathrm{MN}$ & 115,733 & $\mathrm{~N}$ & Y & $\mathrm{N}$ \\
\hline University of Missouri-Columbia & Columbia & $\mathrm{MO}$ & 121,717 & $\mathrm{~N}$ & $\mathrm{~N}$ & Y \\
\hline $\begin{array}{l}\text { University of Mississippi School } \\
\text { of Medicine }\end{array}$ & Jackson & MS & 166,965 & Y & Y & $\mathrm{N}$ \\
\hline $\begin{array}{l}\text { Mary Hitchcock Memorial Hospital/ } \\
\text { Dartmouth-Hitchcock }\end{array}$ & Lebanon & $\mathrm{NH}$ & 13,522 & $\mathrm{~N}$ & Y & $\mathrm{N}$ \\
\hline $\begin{array}{l}\text { University of New Mexico School } \\
\text { of Medicine }\end{array}$ & Albuquerque & NM & 558,545 & $\mathrm{~N}$ & Y & Y \\
\hline $\begin{array}{l}\text { Icahn School of Medicine at } \\
\text { Mount Sinai }\end{array}$ & New York & NY & $8,622,698$ & $\mathrm{~N}$ & $\mathrm{~N}$ & Y \\
\hline $\begin{array}{l}\text { New York Presbyterian Hospital } \\
\text { (Cornell Campus) }\end{array}$ & New York & NY & $8,622,698$ & $\mathrm{~N}$ & Y & $\mathrm{N}$ \\
\hline
\end{tabular}




\begin{tabular}{|c|c|c|c|c|c|c|}
\hline Program name & City & State & $\begin{array}{l}\text { Population }^{\text {b }} \\
\text { (associated } \\
\text { metropolitan area, } \\
\text { if applicable) }\end{array}$ & $\begin{array}{l}\text { Rural } \\
\text { track }\end{array}$ & $\begin{array}{l}\text { Optional } \\
\text { elective } \\
\text { opportunity }\end{array}$ & Teledermatology \\
\hline $\begin{array}{l}\text { Stony Brook Medicine/ } \\
\text { University Hospital }\end{array}$ & Stony Brook & NY & $13,740(8,622,698)$ & $\mathrm{N}$ & Y & $\mathrm{N}$ \\
\hline Duke University Hospital & Durham & NC & 267,743 & $\mathrm{~N}$ & Y & Y \\
\hline University of North Carolina Hospitals & Chapel Hill & NC & 59,862 & $\mathrm{~N}$ & Y & $\mathrm{N}$ \\
\hline $\begin{array}{l}\text { Wake Forest University School } \\
\text { of Medicine }\end{array}$ & $\begin{array}{l}\text { Winston- } \\
\text { Salem }\end{array}$ & NC & 244,605 & $\mathrm{~N}$ & Y & $\mathrm{N}$ \\
\hline Cleveland Clinic Foundation & Cleveland & $\mathrm{OH}$ & 385,525 & $\mathrm{~N}$ & Y & N \\
\hline Ohio State University Hospital & Gahanna & $\mathrm{OH}$ & 35,297 $(787,033)$ & $\mathrm{N}$ & & $\mathrm{N}$ \\
\hline $\begin{array}{l}\text { University of Cincinnati Medical } \\
\text { Center/College of Medicine }\end{array}$ & Cincinnati & $\mathrm{OH}$ & 301,301 & N & & $\mathrm{N}$ \\
\hline $\begin{array}{l}\text { Penn State Milton S Hershey } \\
\text { Medical Center }\end{array}$ & Hershey & PA & 14,257 & $\mathrm{~N}$ & Y & $\mathrm{N}$ \\
\hline $\begin{array}{l}\text { Sidney Kimmel Medical College at } \\
\text { Thomas Jefferson University/TJUH }\end{array}$ & Philadelphia & PA & $1,580,863$ & $\mathrm{~N}$ & Y & $\mathrm{N}$ \\
\hline $\begin{array}{l}\text { University of Pennsylvania } \\
\text { Health System }\end{array}$ & Philadelphia & PA & $1,580,863$ & $\mathrm{~N}$ & Y & $\mathrm{N}$ \\
\hline UPMC Medical Education & Pittsburgh & DA & 302,407 & $\mathrm{~N}$ & Y & Y \\
\hline Brown University & Providence & 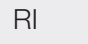 & 47,600 & $\mathrm{~N}$ & Y & $\mathrm{N}$ \\
\hline University of Tennessee & Memphis & $\mathrm{TN}$ & 652,236 & $\mathrm{~N}$ & Y & $\mathrm{N}$ \\
\hline Baylor University Medical Center & & TX & $1,341,075$ & $\mathrm{~N}$ & Y & $\mathrm{N}$ \\
\hline Medical City Weatherford & Weatherford & TX & 30,654 & $\mathrm{~N}$ & Y & $\mathrm{N}$ \\
\hline $\begin{array}{l}\text { Texas A\&M College of Medicine- } \\
\text { Scott and White Medical Center } \\
\text { (Temple) }\end{array}$ & Temple & $\mathrm{TX}$ & 74,503 & $\mathrm{~N}$ & Y & $\mathrm{N}$ \\
\hline $\begin{array}{l}\text { Texas Tech University Health } \\
\text { Sciences Center at Lubbock }\end{array}$ & Lubbock & $\mathrm{TX}$ & 253,888 & $\mathrm{~N}$ & Y & Y \\
\hline $\begin{array}{l}\text { University of Texas Medical } \\
\text { Branch Hospitals }\end{array}$ & Galveston & $\mathrm{TX}$ & 50,497 & $\mathrm{~N}$ & Y & Y \\
\hline OPTI West (Springville Dermatology) & Springville & UT & 33,294 & $\mathrm{~N}$ & Y & $\mathrm{N}$ \\
\hline University of Vermont Medical Center & Burlington & $\mathrm{VT}$ & 42,239 & $\mathrm{~N}$ & Y & $\mathrm{N}$ \\
\hline $\begin{array}{l}\text { University of Wisconsin Hospitals } \\
\text { and Clinics }\end{array}$ & Madison & WI & 255,214 & $\mathrm{~N}$ & Y & Y \\
\hline
\end{tabular}

Abbreviations: ACGME, Accreditation Council for Graduate Medical Education; N, no; Y, yes; NA, not available.

aACGME accreditation status is current through November 12, 2018 at https://apps.acgme.org/ads/Public/Programs/Search.

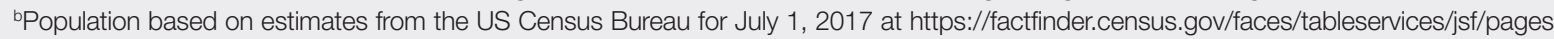
/productview.xhtml?src=bkmk.

'Teledermatology and teledermoscopy within curriculum. 